\title{
Time-Bounded Reachability in Distributed Input/Output Interactive Probabilistic Chains
}

\author{
Georgel Calin ${ }^{1}$, Pepijn Crouzen ${ }^{1}$, Pedro R. D'Argenio ${ }^{2}$, E. Moritz Hahn ${ }^{1}$, and \\ Lijun Zhang $^{3}$ \\ 1 Department of Computer Science, Saarland University, Saarbrücken, Germany \\ 2 FaMAF, Universidad Nacional de Córdoba, Córdoba, Argentina \\ 3 DTU Informatics, Technical University of Denmark, Denmark
}

\begin{abstract}
We develop an algorithm to compute timed reachability probabilities for distributed models which are both probabilistic and nondeterministic. To obtain realistic results we consider the recently introduced class of (strongly) distributed schedulers, for which no analysis techniques are known.

Our algorithm is based on reformulating the nondeterministic models as parametric ones, by interpreting scheduler decisions as parameters. We then apply the PARAM tool to extract the reachability probability as a polynomial function, which we optimize using nonlinear programming.
\end{abstract}

Key words: Distributed Systems, Probabilistic Models, Nondeterminism, Time-Bounded Reachability

\section{Introduction}

This paper considers the computation of reachability probabilities for compositional models with probabilistic and nondeterministic behavior. Such models arise, for instance, in the field of distributed algorithms, where probabilistic behavior is often used to break symmetries in the system. Nondeterminism may appear through the uncertain order of events occurring in different processes or to model freedom of design or unspecified behavior within a process.

Traditional analysis techniques for probabilistic models with nondeterminism compute the maximal and minimal probability to reach a set of configurations by considering all possible resolutions of the nondeterminism [2]. Recently it has been shown that this approach may lead to unrealistic results for models of distributed systems or algorithms [10]. The problem is that the traditional approach allows processes to use non-local information to influence its decisions.

As a running example we will use a simple coin-flip experiment. One player repeatedly flips a coin, while a second player (nondeterministically) guesses the outcome (See Fig. 1). We are interested in the probability that the second player manages to guess correctly at least once within $t$ rounds. Intuitively this probability is $1-\left(\frac{1}{2}\right)^{t}$, but it has been shown that standard analysis methods will produce a probability of 1 for any $t>0$ [10]. The issue is that, from a global point of view, the optimal resolution of the nondeterministic guess simply uses the outcome of the coin-flip as a guide and this way always guesses correctly. 
Distributed schedulers restrict the resolution of nondeterminism by enforcing that local decisions of the processes are based only on local knowledge. For our example, this means that the guesser is not allowed to base the guess on the outcome of the coin-flip. We will see that this leads to realistic results for the probability of attaining a correct guess. Strongly distributed schedulers, in addition, ensure that the relative probability of choosing between two different components does not change with time, provided these components remain idle and uninformed of the progress of the rest of the system.

When considering distributed (or strongly distributed) schedulers, bounds for reachability probabilities are both undecidable and unapproximable in general [9]. However time-bounded reachability probabilities, i.e., the probability to reach a set of configurations within a specified time-period, can be computed. For distributed schedulers, this is due to the fact that optimal solutions in this setting can be computed by only taking into account the subset of deterministic distributed schedulers, which is finite if the system under consideration is finite and acyclic. Nonetheless, the theoretical complexity of this problem is exponential in the number of states. The case of strongly distributed schedulers turns to be more difficult. In this setting, optimal solutions may lie on pure probabilistic schedulers [10]. Therefore, exploring all possible solutions is not an option.

In this paper, we propose to reduce the problem of computing time-bounded reachability probabilities for distributed, probabilistic, and nondeterministic models, under distributed (or strongly distributed) schedulers to a nonlinear optimization problem. We use as our modeling vehicle the formalism of Input/Output Interactive Probabilistic Chains (see Section 2). The computation of time-bounded reachability probabilities is achieved by reformulating the models as Parametric Markov Chains (see Section 5), where the parameters are the decisions of the schedulers and the distributed model is unrolled up to the specified time-point (see Section 6). The time-bounded reachability probability can now be expressed as a polynomial function and we can then compute bounds for it by optimizing the polynomial function under certain constraints.

While for distributed schedulers the only restriction on the variables of the polynomials is that appropriately grouped they form a distribution (i.e. all variables take values between 0 and 1 and each group of variables sum up to 1 ), the case of strongly distributed schedulers require some additional and more complex restrictions, the optimal value of the property being calculated through more involved nonlinear programming techniques.

\section{Input/Output Interactive Probabilistic Chains}

Interactive probabilistic chains (IPCs) are state-based models that combine discrete-time Markov Chains and labelled transition systems [6]. IPCs can be used to compositionally model probabilistic systemss. An important feature of the IPC formalism is that probabilistic transitions and action-labeled transitions are handled orthogonally. As our modeling vehicle we use Input/Output Interactive Probabilistic Chains (I/O-IPCs), a restricted variant of IPCs with a strict separation between local and non-local behavior. The restriction of IPCs to I/O-IPCs follows the one of Interactive Markov Chains to I/O-IMCs in the 
continuous-time setting [3]. The separation between local and non-local behavior is achieved by partitioning the I/O-IPC actions in input, output, and internal actions. In this section we briefly introduce the necessary I/O-IPC definitions.

Let $\operatorname{Dist}(X)$ be the set of all probability distributions over the finite set $X$.

Definition 1. A basic I/O-IPC is a quintuple $\langle S, A, \rightarrow, \Rightarrow, \hat{s}\rangle$ :

$-S$ is a finite set of states with $\hat{s} \in S$ the initial state;

- $A=A^{I} \cup A^{O} \cup A^{\text {int }}$ is a finite set of actions, consisting of disjoint sets of input actions $\left(A^{I}\right)$, output actions $\left(A^{O}\right)$, and internal actions $\left(A^{\text {int }}\right)$;

$-\rightarrow \subseteq S \times A \times S$ is the set of interactive transitions;

- $\Rightarrow: S \rightarrow \operatorname{Dist}(S)$ is a $\mathrm{PF}$ representing the set of probabilistic transitions;

$-\hat{s} \in S$ is the initial state of the I/O-IPC.

Input actions are suffixed by "?", output actions by "!" and we require that an $I / O-I P C$ is both input-enabled, i.e. for each state $s$ and each input action a there is at least one state $s^{\prime}$ such that $\left(s, a, s^{\prime}\right) \in \rightarrow$. We also require that the I/O-IPC is action-deterministic, that is, for each state $s$ and each action a there is at most one state $s^{\prime}$ such that $\left(s, a, s^{\prime}\right) \in \rightarrow$. The non-determinism then stems from the choice between different actions. Finally we require that every state has at least one outgoing, internal, output, or probabilistic transition.

We say that an I/O-IPC is closed if it has no input actions, i.e., $A^{I}=\emptyset$. Note that the requirement of action-determinism is introduced only to simplify the theoretical framework around schedulers. Non-deterministic choices between input transitions can be handled in a similar way as nondeterministic choices between output or internal transitions [10].

Given an action $a$, we use the shorthand notation $s \stackrel{a}{\rightarrow} \mathcal{P} s^{\prime}$ for an interactive transition $\left(s, a, s^{\prime}\right) \in \rightarrow \mathcal{P}$ of $\mathcal{P}$. Given a distribution $\mu$ over the states of $\mathcal{P}$ we use the shorthand notation $s \Rightarrow_{\mathcal{P}} \mu$ for $(s, \mu) \in \Rightarrow_{\mathcal{P}}$. We often leave out the subscript when it is clear from the context.
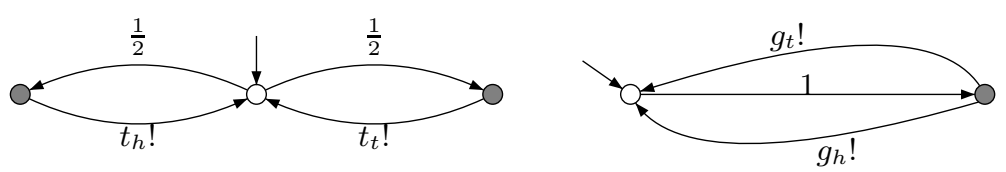

Fig. 1: Basic I/O-IPC Models of the Repeated Coin-Flip Experiment.

The repeated coin-flip (e.g.) is described by the two basic I/O-IPCs in Fig. 1. The coin-flip is depicted on the left-hand side and the coin-toss on the right-hand side. Initial states are indicated by incoming arrows; interactive transitions are labelled with their actions and probabilistic transitions $s \Rightarrow \mu$ are depicted by arrows from $s$ to the support of $\mu$, where each such arrow is labelled with the associated probability. 


\subsection{Parallel composition}

Distributed I/O-IPCs are obtained through parallelizing ( "||") simpler I/O-IPCs.

Definition 2. Two I/O-IPCs $\mathcal{P}$ and $\mathcal{Q}$ are composable if $A_{\mathcal{P}}^{O} \cap A_{\mathcal{Q}}^{O}=A_{\mathcal{P}} \cap$ $A_{\mathcal{Q}}^{\text {int }}=A_{\mathcal{P}}^{\text {int }} \cap A_{\mathcal{Q}}=\emptyset$. If $\mathcal{P}$ and $\mathcal{Q}$ are composable then $\mathcal{C}:=\mathcal{P} \| \mathcal{Q}$ will be

$$
\left\langle S_{\mathcal{P}} \times S_{\mathcal{Q}}, A_{\mathcal{C}}^{I} \cup A_{\mathcal{C}}^{O} \cup A_{\mathcal{C}}^{i n t}, \rightarrow_{\mathcal{C}}, \Rightarrow_{\mathcal{C}},\left(\hat{s}_{\mathcal{P}}, \hat{s}_{\mathcal{Q}}\right)\right\rangle,
$$

where $A_{\mathcal{C}}^{O}:=A_{\mathcal{P}}^{O} \cup A_{\mathcal{Q}}^{O}, A_{\mathcal{C}}^{I}:=\left(A_{\mathcal{P}}^{I} \cup A_{\mathcal{Q}}^{I}\right) \backslash A_{\mathcal{C}}^{O}, A_{\mathcal{C}}^{\text {int }}=A_{\mathcal{P}}^{\text {int }} \cup A_{\mathcal{Q}}^{\text {int }}$ and the transition relations are

$$
\begin{aligned}
\rightarrow_{\mathcal{C}} & =\left\{(s, t) \stackrel{a}{\rightarrow} \mathcal{C}\left(s^{\prime}, t\right) \mid s \stackrel{a}{\rightarrow} \mathcal{P} s^{\prime}, a \in A_{\mathcal{P}} \backslash A_{\mathcal{Q}}\right\} \\
& \cup\left\{(s, t) \stackrel{a}{\rightarrow} \mathcal{C}\left(s, t^{\prime}\right) \mid t \stackrel{a}{\rightarrow} \mathcal{Q} t^{\prime}, a \in A_{\mathcal{Q}} \backslash A_{\mathcal{P}}\right\} \\
& \cup\left\{(s, t) \stackrel{a}{\rightarrow} \mathcal{C}\left(s^{\prime}, t^{\prime}\right) \mid s \stackrel{a}{\rightarrow} \mathcal{P} s^{\prime}, t \stackrel{a}{\rightarrow} t^{\prime}, a \in A_{\mathcal{P}} \cap A_{\mathcal{Q}}\right\} \\
\Rightarrow_{\mathcal{C}} & =\left\{(s, t) \stackrel{\Rightarrow}{\mathcal{C}}_{\mathcal{C}}\left(\mu_{s} \times \mu_{t}\right) \mid s \Rightarrow_{\mathcal{P}} \mu_{s} \wedge t \Rightarrow_{\mathcal{Q}} \mu_{t}\right\}
\end{aligned}
$$

with $\mu_{s} \times \mu_{t}$ denoting the product distribution on $S_{\mathcal{P}} \times S_{\mathcal{Q}}$.

Parallel composition can be extended to any finite set $\mathcal{C}$ of I/O-IPCs in the usual way. Let $\# \mathcal{C}$ denote the number of components of $\mathcal{C}$.

The result of synchronizing an input action with an output action through I/OIPC parallelization will be an output action in the resulting model. As an example, the composition of the basic I/O-IPCs of Fig. 1 is depicted in Fig. 2.

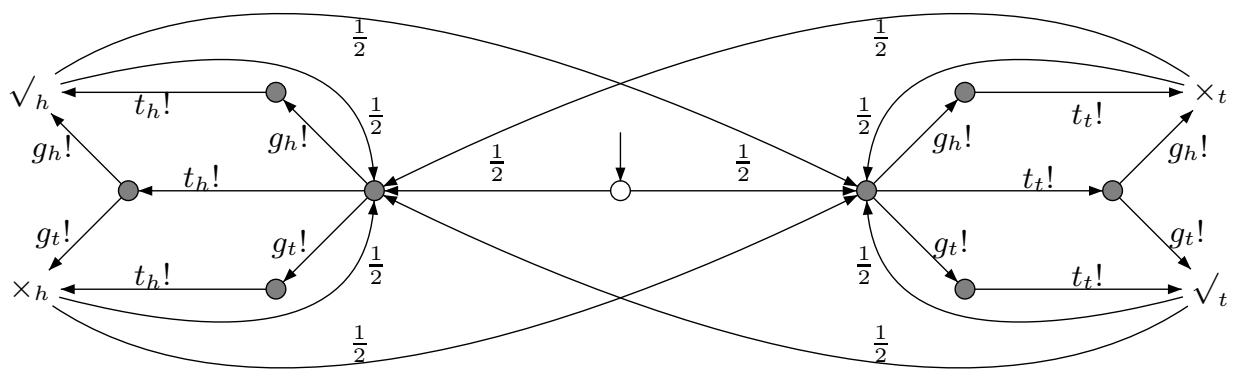

Fig. 2: Distributed I/O-IPC Model of the Repeated Coin-Flip Experiment.

\subsection{Vanishing and tangible states}

The use of distinct probabilistic and instantaneous transitions separates the concerns of time and interaction. In essence, it allows us to specify interactions between components which are instantaneous and do not have to be modeled with explicit time steps. We say that internal and output transitions are immediate and that probabilistic transitions are timed. We now assume that immediate transitions always take precedence over timed transitions. This assumption is known as the maximal progress assumption [14]. This separation between immediate and timed transitions is also reflected in the system states. 
Definition 3 (Vanishing/Tangible States). A state is called vanishing if at least one outgoing immediate transition is enabled in it. If only probabilistic actions are enabled in a state then it is called tangible.

The gray-colored nodes in Figures 1 and 2 are vanishing states, while the rest are all tangible states. For simplicity, in our current study we consider only models that do not exhibit Zeno behaviour (i.e. loops consisting of only immediate actions are not reachable/present in the distributed model).

\subsection{Paths}

An I/O-IPC path describes one possible run of the I/O-IPC. In such a run, we start in a particular state, follow a transition to another state, and so forth.

Definition 4. Given an $I / O-I P C \mathcal{P}=\langle S, A, \rightarrow, \Rightarrow, \hat{s}\rangle$, a finite path of $\mathcal{P}$ of length $n \in \mathbb{N}$ is a sequence $s_{0} a_{0} s_{1} a_{1} \ldots a_{n-1} s_{n}$ where states $\left(s_{i} \in S\right.$ for $\left.i \in[0, n]\right)$ and either actions or distributions ( $a_{i} \in A \cup \operatorname{Dist}(S)$, for $i \in[0, n-1]$ ) are interleaved. For consecutive states $s_{i}$ and $s_{i+1}$ we find that either $a_{i} \in A$ and $\left(s_{i}, a_{i}, s_{i+1}\right) \in \rightarrow$ or $a_{i} \in \operatorname{Dist}(S), s_{i}$ is tangible, $\left(s_{i}, a_{i}\right) \in \Rightarrow$, and $a_{i}\left(s_{i+1}\right)>0$. An infinite path of $\mathcal{P}$ is an infinite sequence $s_{0} a_{0} s_{1} a_{1} \ldots$ interleaving states and actions/distributions. We denote the last state of a finite path $\sigma$ as last $(\sigma)$.

For studying time-bounded reachability, we need a notion of time. We follow the definition of time in IPCs and say that only probabilistic transitions take time, while interactive transitions are considered to take place immediately [5].

Definition 5. The elapsed time along a finite path $\sigma$, notation $t(\sigma)$, is defined recursively, for states $s$, actions $a$ and distributions $\mu$ over states: $\mathrm{t}(s)=0$, $\mathrm{t}(\sigma a s)=t(\sigma)$, and $\mathrm{t}(\sigma \mu s)=t(\sigma)+1$.

\subsection{Schedulers}

We now wish to associate paths of an I/O-IPC with probabilities. The usual strategy is to define the probability of a path as the multiplication of the probabilities of its transitions. To define such a probability for paths in an I/O-IPC we need some way of resolving the non-deterministic choice between interactive transitions in vanishing states of an I/O-IPC. For all states $s \in S$, let $A_{s, \mathcal{P}}^{e n}=\left\{a \in A^{O} \mid \exists s^{\prime} . s \stackrel{a}{\rightarrow} s^{\prime}\right\} \cup\left\{a \in A^{i n t} \mid \exists s^{\prime} . s \stackrel{a}{\rightarrow} s^{\prime}\right\}$ be the set of enabled immediate actions for $s$.

Definition 6. A scheduler for an I/O-IPC $\mathcal{P}$ is the function $\eta_{\mathcal{P}}:$ Paths $(\mathcal{P}) \rightarrow$ $\operatorname{Dist}\left(A_{\mathcal{P}}\right)$, such that positive probability are assigned only to actions enabled in the last state of a path: $\eta_{\mathcal{P}}(\sigma)(a)>0$ implies that $a \in A_{\text {last }(\sigma), \mathcal{P}}^{\text {en }}$

If $\mathcal{P}$ is closed, then a scheduler determines the probability to observe a certain path, which also allows us to define time-bounded reachability probabilities. We give the details, in the context of distributed schedulers, in Section 4. 


\section{Distributed Schedulers}

As we have seen, the probability of reaching a set of goal states in a distributed I/O-IPC depends on how the nondeterminism of chosing an action is handled. By assigning probabilities to the available actions, a scheduler can be seen as a refinement of the I/O-IPC such that the induced model becomes deterministic. It can thus be said that a scheduler enables us to determine reachability probabilities in a deterministic fashion.

However, the class of all schedulers for the model of a distributed system contains schedulers that are unrealistic in that they allow components of the system to use non-local information to guide their local decisions. To overcome this problem, distributed schedulers have been introduced, that restrict the possible choices of a scheduler to make them more realistic in a distributed setting [10]. Distributed schedulers have originally been introduced for (switched) probabilistic input/output automata [2] and we adapt them here for our Input/Output Interactive Markov Chains formalism.

To illustrate the necessity of distributed schedulers, consider the game described in Fig. 2 where an unbiased coin is repeatedly tossed and guessed by two independent entities at the same time. We are interested in the probability to reach the set of states labelled $\sqrt{ }$ within a specified number $t$ of timed(probabilistic) steps. This is exactly the probability that the guessing player guesses correctly within at most $t$ tries. Intuitively, for each matching toss/guess, since the tossing player makes its choice probabilistically and the guessing player does not observe the outcome, the guessing player should have a probability of one half of making the right guess and winning the game.

However, it is clear that in the composed model there is a scheduler that arrives with probability one at a $\sqrt{ }$ state within at most one timed step. This scheduler simply chooses the action $t_{h}$ if heads is tossed and $t_{t}$ if tails is tossed, thereby always winning. The purpose of distributed schedulers is to make sure that the decision between $t_{h}$ and $t_{t}$ is made only based on local information.

\subsection{Distributed Schedulers}

The main principle of distributed schedulers is to use a separate scheduler for each of the components of the system such that each has access only to their own scheduling history. To be able to reason about local information we first introduce path projections.

Given any distributed I/O-IPC $\mathcal{C}=\mathcal{P}_{1}\|\ldots\| \mathcal{P}_{n}$ and a path $\sigma \in \operatorname{Paths}(\mathcal{C})$, the projection $\sigma\left[\mathcal{P}_{i}\right]$ of $\sigma$ on $\mathcal{C}$ 's $i$-th basic component is given by:

$$
\begin{aligned}
& -\left(\hat{s}_{\mathcal{C}}\right)\left[\mathcal{P}_{i}\right]=\pi_{i}\left(\hat{s}_{\mathcal{C}}\right) \\
& -(\sigma a s)\left[\mathcal{P}_{i}\right]= \begin{cases}\sigma\left[\mathcal{P}_{i}\right] & \text { if } a \notin A_{\mathcal{P}_{i}} \\
\left(\sigma\left[\mathcal{P}_{i}\right]\right) a\left(\pi_{i}(s)\right) & \text { if } a \in A_{\mathcal{P}_{i}}\end{cases} \\
& -\left(\sigma\left(\mu_{1} \times \cdots \times \mu_{n}\right) s\right)\left[\mathcal{P}_{i}\right]=\left(\sigma\left[\mathcal{P}_{i}\right]\right) \mu_{i}\left(\pi_{i}(s)\right) .
\end{aligned}
$$

where $\pi_{i}\left(\left(s_{1}, \ldots, s_{n}\right)\right)=s_{i}$ for all $\left(s_{1}, \ldots, s_{n}\right) \in S_{\mathcal{C}}$.

A local scheduler for $\mathcal{P}$ is simply any scheduler for $\mathcal{P}$ as given by Def. 6 . A local scheduler resolves the nondeterminism arising from choices between enabled 
output and internal actions in one of the components. However, nondeterminism may also arise from the interleaving of the different components. In other words, if for some state in a distributed I/O-IPC, two or more components have enabled immediate actions, then it must be decided which component acts first. This decision is made by the interleaving scheduler.

Definition 7. Given a distributed I/O-IPC C $=\mathcal{P}_{1}\|\ldots\| \mathcal{P}_{n}$, an interleaving scheduler $\mathcal{I}:$ Paths $(\mathcal{C}) \rightarrow \operatorname{Dist}\left(\left\{\mathcal{P}_{1}, \ldots, \mathcal{P}_{n}\right\}\right)$ is defined for paths $\sigma$ such that last $(\sigma)$ is vanishing. The interleaving scheduler $\mathcal{I}$ chooses probabilistically an enabled component of the distributed system, i.e., we have that $\mathcal{I}(\sigma)\left(\mathcal{P}_{i}\right)>0$ implies $A_{\text {last }\left(\sigma\left[\mathcal{P}_{i}\right]\right), \mathcal{P}_{i}}^{\text {en }} \neq \varnothing$.

Local schedulers and an interleaving scheduler form a distributed scheduler.

Definition 8. Given a distributed I/O-IPC $\mathcal{C}=\mathcal{P}_{1}\|\ldots\| \mathcal{P}_{n}$, local schedulers $\eta_{\mathcal{P}_{1}}, \ldots, \eta_{\mathcal{P}_{1}}$, and interleaving scheduler $\mathcal{I}$, the associated distributed scheduler is the function $\eta_{\mathcal{C}}:$ Paths $(\mathcal{C}) \rightarrow \operatorname{Dist}\left(A_{\mathcal{C}}\right)$ such that, for all $\sigma \in$ Paths $(\mathcal{C})$ with last $(\sigma)$ vanishing and for all $a \in A_{\mathcal{C}}$ :

$$
\eta_{\mathcal{C}}(\sigma)(a)=\sum_{i=1}^{n} \mathcal{I}(\sigma)\left(\mathcal{P}_{i}\right) \cdot \eta_{\mathcal{P}_{i}}\left(\sigma\left[\mathcal{P}_{i}\right]\right)(a)
$$

We denote the set of all distributed schedulers as $D S$.

\subsection{Strongly Distributed Schedulers}

Although the class of distributed schedulers already realistically restricts the local decisions of processes in a distributed setting, in certain cases there exist distributed schedulers, where the interleaving schedulers are to powerful. In essence, the problem is that a distributed scheduler may use information from a component $P_{1}$ to decide how to pick between components $P_{2}$ and $P_{3}$. In certain settings this is unrealistic. To counter this problem strongly distributed schedulers have been introduced [10].

Given any two components $P_{i}, P_{j}$ of a distributed I/O-IPC $C=P_{1}\|\ldots\| P_{n}$, consider the following property: for all $\sigma, \sigma^{\prime}$ such that $\sigma\left[P_{i}\right]=\sigma^{\prime}\left[P_{i}\right]$ and $\sigma\left[P_{j}\right]=$ $\sigma^{\prime}\left[P_{j}\right]$, if $\mathcal{I}(\sigma)\left(P_{i}\right)+\mathcal{I}(\sigma)\left(P_{j}\right) \neq 0$ and $\mathcal{I}\left(\sigma^{\prime}\right)\left(P_{i}\right)+\mathcal{I}\left(\sigma^{\prime}\right)\left(P_{j}\right) \neq 0$ then

$$
\frac{\mathcal{I}(\sigma)\left(P_{i}\right)}{\mathcal{I}(\sigma)\left(P_{i}\right)+\mathcal{I}(\sigma)\left(P_{j}\right)}=\frac{\mathcal{I}\left(\sigma^{\prime}\right)\left(P_{i}\right)}{\mathcal{I}\left(\sigma^{\prime}\right)\left(P_{i}\right)+\mathcal{I}\left(\sigma^{\prime}\right)\left(P_{j}\right)} .
$$

Definition 9. A scheduler $\eta$ is strongly distributed if it is distributed and the restriction in Eq. (1) holds for the interleaving scheduler $\mathcal{I}$ of $\eta$.

The intuition behind strongly distributed scheduler is that the choices the interleaving scheduler makes between two components $P_{i}, P_{j}$ should be consistent with respect to the local paths of $P_{i}, P_{j}$. If for two global paths, the local paths of $P_{i}, P_{j}$ are identical, then the probability of choosing $P_{i}$ under the condition that we choose either $P_{i}$ or $P_{j}$ should be identical for both global paths. 
Strongly distributed schedulers are useful depending on which system is considered for study [10]. When analyzing an auctioning protocol, for example, where each component models one of the bidders, then the order in which the bidders interact with the auctioneer should not leak information that can be used to the advantage of the other bidders. In such a situation, strongly distributed schedulers would provide more adequate worst-case/best-case probabilities.

However, if the interleaving scheduler should have access to the history of the components (as it might be the case for a kernel scheduler on a computer) then distributed schedulers should be considered, as the strongly distributed version might rule out valid possibilities.

We denote the set of all distributed schedulers as $S D S$.

\section{Induced Probability Measure}

When all the non-deterministic choices in a distributed I/O-IPC are resolved by a scheduler, we end up with a probability measure on sets of paths of the I/O-IPC. We define this probability measure in a similar way as is done for IPCs [15]. We fix a closed distributed I/O-IPC $\mathcal{C}=\mathcal{P}_{1}\|\ldots\| \mathcal{P}_{n}$ with state space $S_{\mathcal{C}}$, actions $A_{\mathcal{C}}$, and initial state $\hat{s}$.

The cylinder induced by the finite path $\sigma$ is the set of infinite paths $\sigma^{\uparrow}=$ $\left\{\sigma^{\prime} \mid \sigma^{\prime}\right.$ is infinite and $\sigma$ is a prefix of $\left.\sigma^{\prime}\right\}$. Let the set of cylinders generate the $\sigma$-algebra on infinite paths of $\mathcal{C}$.

Definition 10. Let $\eta$ be a (possibly strongly) distributed scheduler on $\mathcal{C}$. The probability measure induced by $\eta$ on the set of infinite paths is the unique probability measure $P_{\eta}$ such that, for any state $s$ in $S_{\mathcal{C}}$, any action a in $A_{\mathcal{C}}$ and any distribution $\mu \in \operatorname{Dist}\left(S_{\mathcal{C}}\right)$ :

$$
\begin{aligned}
P_{\eta}\left(s^{\uparrow}\right) & = \begin{cases}1 & \text { if } s=\hat{s} \\
0 & \text { otherwise }\end{cases} \\
P_{\eta}\left(\sigma a s^{\uparrow}\right) & = \begin{cases}P_{\eta}\left(\sigma^{\uparrow}\right) \cdot \eta(\sigma)(a) & \text { if last }(\sigma) \text { is vanishing and last }(\sigma) \stackrel{a}{\longrightarrow} s \\
0 & \text { otherwise }\end{cases} \\
P_{\eta}\left(\sigma \mu s^{\uparrow}\right) & = \begin{cases}P_{\eta}\left(\sigma^{\uparrow}\right) \cdot \mu(s) & \text { if last }(\sigma) \text { is tangible and last }(\sigma) \Rightarrow \mu \\
0 & \text { otherwise }\end{cases}
\end{aligned}
$$

We are now ready to define time-bounded reachability for I/O-IPCs.

Definition 11. Given an I/O-IPC $\mathcal{P}$ with an initial distribution, a set of goal states $\mathcal{G}$ and a time-bound $t \in \mathbb{N}$, we have that the probability to reach $\mathcal{G}$ within $t$ time-steps, denoted $P_{\eta}\left(\diamond^{\leq t} \mathcal{G}\right)$, is:

$$
P_{\eta}\left(\diamond^{\leq t} \mathcal{G}\right)=P_{\eta}\left(\bigcup\left\{\sigma^{\uparrow} \mid t(\sigma) \leq t \text { and last }(\sigma) \in \mathcal{G}\right\}\right)
$$

\section{Parametric Markov Models}

To compute time-bounded reachability probabilities we will transform distributed I/O-IPCs into Parametric Markov models (see Section 6). In this section we give a brief overview of parametric Markov Chains [7, 12,11]. First, we introduce some general notations. 
Let $S$ be a finite set of states. We let $V=\left\{x_{1}, \ldots, x_{n}\right\}$ denote a set of variables with domain $\mathbb{R}$. An assignment $\zeta$ is a function $\zeta: V \rightarrow \mathbb{R}$. A polynomial $g$ over $V$ is a sum of monomials $g\left(x_{1}, \ldots, x_{n}\right)=\sum_{i_{1} \ldots, i_{n}} a_{i_{1}, \ldots, i_{n}} x_{1}^{i_{1}} \cdots x_{n}^{i_{n}}$ where each $i_{j} \in \mathbb{N}_{0}$ and each $a_{i_{1}, \ldots, i_{n}} \in \mathbb{R}$. A rational function $f$ over a set of variables $V$ is a fraction $f\left(x_{1}, \ldots, x_{n}\right)=f_{1}\left(x_{1}, \ldots, x_{n}\right) / f_{2}\left(x_{1}, \ldots, x_{n}\right)$ of two polynomials $f_{1}, f_{2}$ over $V$.

Let $\mathcal{F}_{V}$ denote the set of rational functions from $V$ to $\mathbb{R}$. Given $f \in \mathcal{F}_{V}$ and an assignment $\zeta$, we let $\zeta(f)$ denote the rational function obtained by substituting each occurrence of $x \in V$ with $\zeta(x)$.

Definition 12. A parametric Markov chain $(P M C)$ is a tuple $\mathcal{D}=(S, \hat{s}, \mathbf{P}, V)$ where $S$ is a finite set of states, $\hat{s}$ is the initial state, $V=\left\{v_{1}, \ldots, v_{n}\right\}$ is a finite set of parameters and $\mathbf{P}$ is the probability matrix $\mathbf{P}: S \times S \rightarrow \mathcal{F}_{V}$.

The matrix $\mathbf{P}$ denotes the probabilities to go from one state to another in one step. We now generalize this for $k$ steps.

Definition 13. Given a $P M C \mathcal{D}=(S, \hat{s}, \mathbf{P}, V)$, the $k$-step probability matrix $\mathbf{P}_{k}, k \in \mathbb{N}$, is defined recursively for any $k^{\prime}>1$ and states $s, s^{\prime} \in S$ :

$$
\begin{aligned}
\mathbf{P}_{0}\left(s, s^{\prime}\right) & =\left\{\begin{array}{l}
1 \text { if } s=s^{\prime} \\
0 \text { if } s \neq s^{\prime}
\end{array}\right. \\
\mathbf{P}_{k^{\prime}}\left(s, s^{\prime}\right) & =\sum_{s^{\prime \prime} \in S} \mathbf{P}_{k^{\prime}-1}\left(s, s^{\prime \prime}\right) \cdot \mathbf{P}\left(s^{\prime \prime}, s^{\prime}\right)
\end{aligned}
$$

\section{Parametric Interpretation}

By having the scheduler $\eta$ fixed, $P_{\eta}$ together with the scheduled I/O IPC $\mathcal{C}$ would become deterministic. The big difference to, e.g., DTMC models is that in our case the model is not memoryless (the scheduler depends on entire paths). However, by treating the interleaving and local scheduler decisions as unknowns we arrive at analyzing parametric Markov Chains, the parameters being precisely the decisions that the interleaving and local schedulers perform.

We have seen in Section 4 that fixing the scheduler of a distributed I/O-IPC induces a probability measure on paths. Our approach is now to fix the scheduler parametrically, i.e., by treating the probabilities chosen by the interleaving and local schedulers as parameters. We will show that this unfolding of the I/O-IPC induces a PMC (see Section 5) whose states are paths of the distributed I/OIPC. To make sure the induced PMC is finite we generate it only for paths up to a specific time-bound $t$. We then prove that computing the probability to reach a set of states within $t$ time-units for the induced PMC is equivalent to computing it for the I/O-IPC.

To give an idea of how this unfolding works, consider again the repeated coin-flip experiment, depicted in Fig. 1 and 2. Intuitively it should hold that $\operatorname{Pr}\left(\diamond^{\leq 2}\left\{\sqrt{ }_{h}, \sqrt{ }_{t}\right\}\right)=3 / 4$ if we assume the guessing player has no information about the outcome of each coin-flip. Fig. 3 describes the unfolding of the distributed I/O-IPC from Figure 2 up to time-point 2. On the right-hand side we 
see the structure of the PMC for one time-step. The unfolding up to 2 time steps is shown schematically on the left-hand side, where each square represents a copy of the structure on the right-hand side.

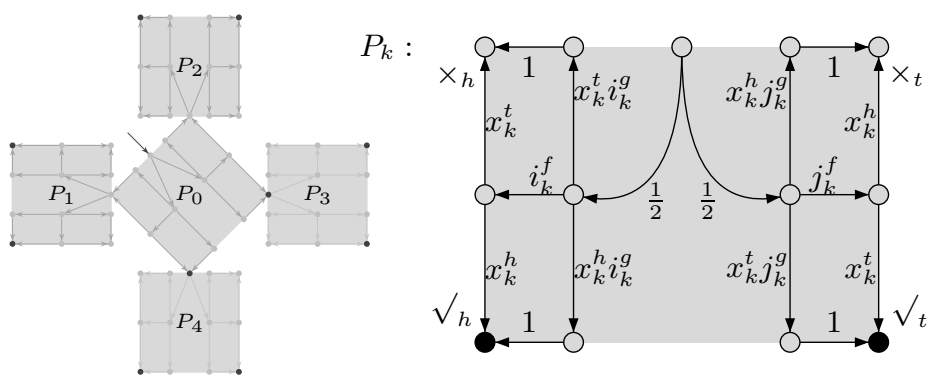

Fig. 3: Repeated Coin-Flip Experiment (PMC-unfolding Scheme up to Time 2)

The local scheduler decisions in this case for each repeating structure $P_{k}$ are $x_{k}^{h}, x_{k}^{t}$ s.t. $x_{k}^{h}+x_{k}^{t}=1$ and the interleaving scheduler decisions are $i_{k}^{g}, i_{k}^{f}, j_{k}^{g}, j_{k}^{f}$ s.t. $i_{k}^{g}+i_{k}^{f}=j_{k}^{g}+j_{k}^{f}=1$. Here $x_{k}^{h}$, for example, denotes the probability assigned by the local scheduler for the guesser to pick "heads" for a local path ending in a "heads" vs. "tail" choice. The parameters $i_{k}^{g}$ and $i_{k}^{f}$ denote the probabilities the interleaving scheduler assigns to the "guessing" model and the "flipping model" respectively, for a given global path which enables them both.

Now, $\operatorname{Pr}\left(\diamond^{\leq 2}\left\{\sqrt{h}_{h}, \sqrt{ }_{t}\right\}\right)$ can be computed as the sum of the cumulated probabilities on the paths leading to $\left\{\sqrt{h}_{h} \cdot \sqrt{ }_{t}\right\}$ states by using the given unfolding in Fig. 3 and the above parameter restrictions:

$\frac{1}{2}\left(x_{0}^{h} \cdot i_{0}^{g}+i_{0}^{f} \cdot x_{0}^{h}+\left(x_{0}^{t} \cdot i_{0}^{g}+i_{0}^{f} \cdot x_{0}^{t}\right) \cdot\left[\frac{1}{2}\left(x_{1}^{h} \cdot i_{1}^{g}+i_{1}^{f} \cdot x_{1}^{h}\right)+\frac{1}{2}\left(x_{1}^{t} \cdot i_{1}^{g}+x_{1}^{t} \cdot i_{1}^{f}\right)\right]\right)+$

$\frac{1}{2}\left(x_{0}^{t} \cdot i_{0}^{g}+i_{0}^{f} \cdot x_{0}^{t}+\left(x_{0}^{h} \cdot i_{0}^{g}+i_{0}^{f} \cdot x_{0}^{h}\right) \cdot\left[\frac{1}{2}\left(x_{2}^{h} \cdot i_{2}^{g}+i_{2}^{f} \cdot x_{2}^{h}\right)+\frac{1}{2}\left(x_{2}^{t} \cdot i_{2}^{g}+i_{2}^{f} \cdot x_{2}^{t}\right)\right]\right)=$ $\frac{1}{2}\left(x_{0}^{h}+x_{0}^{t} \cdot\left(\frac{1}{2} x_{1}^{h}+\frac{1}{2} x_{1}^{t}\right)\right)+\frac{1}{2}\left(x_{0}^{t}+x_{0}^{h} \cdot\left(\frac{1}{2} x_{2}^{h}+\frac{1}{2} x_{2}^{t}\right)\right)=\frac{3}{4}$

We now define the above interpretation of scheduler decisions as parameters formally.

Definition 14. Let $S_{\mathcal{C}}^{t} \subseteq$ Paths $(\mathcal{C})$ be the set of all paths in a closed, distributed $I / O-I P C \mathcal{C}=\mathcal{P}_{1}\|\ldots\| \mathcal{P}_{n}$, with time-length $\leq t$. Define the parameters set $V$ by

$$
\begin{aligned}
V= & \left\{y_{\sigma}^{i} \mid \sigma \in S_{\mathcal{C}}^{t}, 1 \leq i \leq \# \mathcal{C}, A_{\text {last }\left(\sigma\left[\mathcal{P}_{i}\right]\right), \mathcal{P}_{i}}^{\text {en }} \neq \varnothing\right\} \cup \\
& \left\{x_{\sigma\left[\mathcal{P}_{i}\right]}^{a} \mid \sigma \in S_{\mathcal{C}}^{t}, 1 \leq i \leq \# \mathcal{C}, a \in A_{\text {last }\left(\sigma\left[\mathcal{P}_{i}\right]\right), \mathcal{P}_{i}}^{\text {en }}\right\}
\end{aligned}
$$

and let $\mathbf{P}$ match the induced probability measure, namely for any path $\sigma \in S_{\mathcal{C}}^{t}$, any state $s$ of $\mathcal{C}$, any action $a$ of $\mathcal{C}$ and any distribution $\mu$ over the states of $\mathcal{C}$ :

$$
\begin{aligned}
\mathbf{P}(\sigma, \sigma a s) & =y_{\sigma}^{i} \cdot x_{\sigma\left[\mathcal{P}_{i}\right]}^{a} & \text { if last }(\sigma) \text { is vanishing, last }(\sigma) \stackrel{a}{\rightarrow} s, a \in A_{\text {last }\left(\sigma\left[\mathcal{P}_{i}\right]\right), \mathcal{P}_{i}}^{\text {en }} \\
\mathbf{P}(\sigma, \sigma \mu s) & =\mu(s) & \text { if last }(\sigma) \text { is tangible, } \mathrm{t}(\sigma)<t, \text { last }(\sigma) \Rightarrow \mu \\
\mathbf{P}(\sigma, \sigma) & =1 & \text { if last }(\sigma) \text { is tangible, } \mathrm{t}(\sigma)=t .
\end{aligned}
$$


All other transition probabilities are zero. The unfolding of the I/O-IPCC up to time bound $t$ is then the $P M C \mathcal{D}=\left(S_{\mathcal{C}}^{t}, \hat{s}_{\mathcal{C}}, \mathbf{P}, V\right)$.

The finiteness of $S_{\mathcal{C}}^{t}$ and $V$ is guaranteed by the exclusion of infinite chains consisting of only immediate actions, as it implies that for each state in $\mathcal{C}$ a tangible state is reachable within a finite number of non-probabilistic steps. The variables in Def. 14 can be restricted to ensure that they represent valid scheduler decisions in the following way:

$$
\begin{array}{ll}
0 \leq v \leq 1 & \text { if } v \in V \\
\sum_{a \in A} x_{\sigma\left[\mathcal{P}_{i}\right]}^{a}=1 & \text { if } \sigma \in S_{\mathcal{C}}^{t} \text { and } 1 \leq i \leq \# \mathcal{C} \text { with } A=A_{\text {last }\left(\sigma\left[\mathcal{P}_{i}\right]\right), \mathcal{P}_{i}}^{\text {en }} \\
\sum_{i \in I} y_{\sigma}^{i}=1 & \text { if } \sigma \in S_{\mathcal{C}}^{t} \text { with } I=\left\{i \mid 1 \leq i \leq \# C \text {, last }\left(\sigma\left[P_{i}\right]\right) \text { vanishing }\right\}
\end{array}
$$

Under these restrictions we can connect path probabilities of I/O-IPCs to $k$-step transition probabilities of the induced PMC.

Lemma 1. For a closed, distributed I/O-IPC C , let $\mathcal{D}$ be as in Def. 14. Then

(i) For all distributed scheduler $\eta$ there is an assignment $\zeta: V \rightarrow[0,1]$ satisfying 2 such that for all $\sigma \in S_{\mathcal{C}}^{t}: P_{\eta}\left(\sigma^{\uparrow}\right)=\zeta\left(\mathbf{P}_{k}(\hat{s}, \sigma)\right)$ where $k$ is the length of $\sigma$.

(ii) Reciprocally, for all assignment $\zeta: V \rightarrow[0,1]$ satisfying (2) there is a distributed scheduler $\eta$ such that for all $\sigma \in S_{\mathcal{C}}^{t}: P_{\eta}\left(\sigma^{\uparrow}\right)=\zeta\left(\mathbf{P}_{k}(\hat{s}, \sigma)\right)$.

The proof of Lemma 1 can be found in Appendix A.

We can now reformulate the bounded reachability problem for I/O-IPCs under distributed schedulers as an unbounded reachability problem for the associated induced PMC.

Theorem 1. Time-bounded reachability for an I/O-IPC $\mathcal{C}$ under distributed schedulers is equivalent to checking time-unbounded reachability on the PMC $\mathcal{D}=\left(S_{\mathcal{C}}^{t}, \hat{s}_{\mathcal{C}}, \mathbf{P}, V\right)$ as in Def. 14 for assignments that satisfy (2):

$$
\sup _{\eta \in D S} P_{\eta}\left(\diamond^{\leq t} \mathcal{G}\right)=\sup _{\zeta \text { sat. (2) }} \zeta\left(P_{\mathcal{D}}(\diamond \mathcal{G})\right) \text { and } \inf _{\eta \in D S} P_{\eta}\left(\diamond^{\leq t} \mathcal{G}\right)=\inf _{\zeta \text { sat. (2) }} \zeta\left(P_{\mathcal{D}}(\diamond \mathcal{G})\right) \text {. }
$$

The proof of Theorem 1 can be found in Appendix A.

To extend this result to strongly distributed schedulers we must further restrict the variables of the induced PMC such that the allwoed assignments match the strongly distributed schedulers. First we introduce new variables which represent the conditional probabilities in (1). For every $i, j, 1 \leq i, j \leq \# \mathcal{C}, i \neq j$, and $\sigma \in S_{\mathcal{C}}^{t}$, define a new variable $z_{\sigma\left[\mathcal{P}_{i}\right], \sigma\left[\mathcal{P}_{j}\right]}^{i, j} \notin V$. Notice that two different $\sigma, \sigma^{\prime} \in S_{\mathcal{C}}^{t}$ may induce the same variable if $\sigma\left[\mathcal{P}_{i}\right]=\sigma^{\prime}\left[\mathcal{P}_{i}\right]$ and $\sigma\left[\mathcal{P}_{j}\right]=\sigma^{\prime}\left[\mathcal{P}_{j}\right]$. We write $V_{z}$ for the set of all such variables $z_{\sigma\left[\mathcal{P}_{i}\right], \sigma\left[\mathcal{P}_{j}\right]}^{i,}$.

Using these new variables we pose new restrictions on the variables in the induced PMC of a distributed I/O-IPC.

$$
z_{\sigma\left[\mathcal{P}_{i}\right], \sigma\left[\mathcal{P}_{j}\right]}^{i, j}\left(y_{\sigma}^{i}+y_{\sigma}^{j}\right)=y_{\sigma}^{i} \quad \text { if } 1 \leq i, j \leq \# \mathcal{C}, i \neq j \text {, and } \sigma \in S_{\mathcal{C}}^{t}
$$


Theorem 2. Time-bounded reachability for an I/O-IPC C under strongly distributed schedulers is equivalent to checking reachability on the parametric Markov model $\mathcal{D}=\left(S_{\mathcal{C}}^{t}, \hat{s}_{\mathcal{C}}, \mathbf{P}, V \cup V_{z}\right)$ resulted through unfolding as in Def. 14 under the assumptions (2) and (3).

The proof of Theorem 2 can be found in Appendix A.

Time-unbounded reachability probabilities for PMCs can be computed using the tool PARAM, which, since our PMC models are acyclic, results in analyzing a set of polynomial functions over the given variables. These polynomial functions can then be optimized under the constraints given by (2) and - for strongly distributed schedulers - (3) using standard numerical solvers. Together, these steps form our algorithm.

\section{Algorithm}

We now present our algorithm to compute extremal time-bounded reachability probabilities for distributed I/O-IPCs. Our algorithm requires the following inputs: a closed, distributed I/O-IPC which exhibits no Zeno-behavior $\mathcal{C}$, a timebound $t$, and a set of goal states $\mathcal{G}$. The following steps are sequentially executed:

1. The I/O-IPC is unfolded up to time-bound $t$, yielding a PMC (as per Def. 14). Additionally linear constraints on the parameters are provided to ensure that all scheduler decisions lie in the interval $[0,1]$ and that specific sets of parameters sum up to 1 . In the case of strongly distributed schedulers, non-linear constraints are also generated as described in Thm. 2.

2. The time-unbounded reachability probability for the set of goal states $\mathcal{G}$ is calculated parametrically for the PMC generated in step 1 using the PARAM tool [11]. The result is a polynomial function.

3 . The polynomial function generated in step 2 is optimized under the constraints generated in step 3 using non-linear programming. We have used the active-set algorithm $[8,13]$ provided by the fmincon function of Mat$\mathrm{lab}^{4}$, but any non-linear programming tool can in principal be used.

An overview of this tool-chain is presented in Fig. 4. The tool which unfolds the I/O-IPC and generates a PMC together with linear and non-linear constraints is still under development. We have, however generated PMC models and constraints semi-automatically for several case studies which we present next.

\section{Case Studies}

In this section we apply our algorithm to three case studies. Since the Unfolder tool, which translates distributed I/O-IPCs into PMCs and constraints, is still under development we have generated PMCs and constraints for these cases in a semi-automatic way. The PARAM tool has been run on a computer with a 3 Ghz processor and $1 \mathrm{~GB}$ of memory, while Matlab was run on a computer with two 1.2 Ghz processors and 2 GB of memory. All I/O-IPC and PMC models are available from the authors.

\footnotetext{
${ }^{4}$ See http://www.mathworks.com
} 


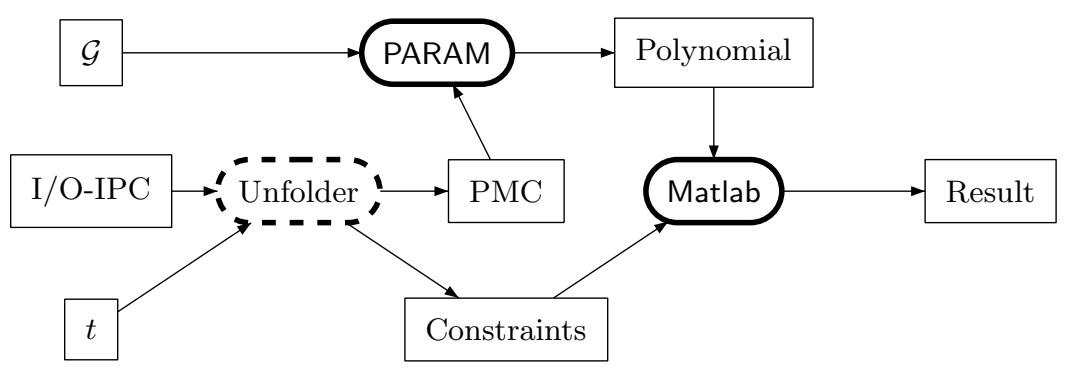

Fig. 4: The Envisioned Tool-Chain: ellipses represent tools, boxes represent data, where dashed ellipses represent tools that are currently in development.

\subsection{Mastermind}

In the game of Mastermind [1] one player, the guesser, tries to find out a code, generated by the other player, the encoder. The code consisting of a number of tokens of fixed positions, where for each token one color (or other labelling) out of a pre-specified set is chosen. Colors can appear multiple times.

Each round, the guesser guesses a code. This code is then compared to the correct one by the encoder. The encoder answers by telling the guesser a) how many tokens were of the correct color and at the correct place and b) how many tokens were not at the correct place, but have a corresponding token of the same color in the code.

Notice that the decisions of the encoder during the game are fully deterministic, while the guesser has the choice between all valid codes. We assume that the encode chooses the code probabilistically with a uniform distribution over all options. The goal of the guesser is to find out the code as fast as possible. Our aim is now to compute the maximal probability that the guesser correctly guesses the code within $t$ rounds.

We formalize the game as follows: we let $n$ denote the number of tokens of the code and let $m$ denote the number of colors. This means there are $m^{n}$ possible codes. Let $\mathcal{O}$ denote the set of all possible codes. We now informally describe the I/O-IPC models which represent the game of Mastermind. The guesses are described by actions $\left\{g_{o} \mid o \in \mathcal{O}\right\}$, whereas the answers are described by actions $\left\{a_{(x, y)} \mid x, y \in[0, n]\right\}$.

The guesser $\mathcal{G}$ repeats the following steps: From the initial state, $s_{\mathcal{G}}$ it first takes a probabilistic step to state $s_{\mathcal{G}}^{\prime}$ and afterwards the guesser returns to the initial state via one of $m^{n}$ transitions, each labelled with an output action $g_{o}$ !. In both states the guesser receives answers $a_{(x, y)}$ ? from the encoder and for all answers the guesser simply remains in the same state, except for the answer $a_{(n, n)}$ which signals that the guesser has guessed correctly. When the guesser receives this action it moves to the absorbing state $s_{\mathcal{G}}^{\prime \prime}$.

The encoder $\mathcal{E}$ is somewhat more complex. It start by picking a code probabilistically, where each code has the same probability $\frac{1}{\mathrm{~m}^{n}}$. Afterwards the encoder repeats the following steps indefinitely. First it receives a guess from the 
guesser, then it replies with the appropriate answer and then it takes a probabilistic transition. This probabilistic step synchronizes with the probabilistic step of the guesser, which allows us to record the number of rounds the guesser needs to find the code.

The Mastermind game is thus the composition $\mathcal{C}:=\mathcal{G} \| \mathcal{E}$ of the two basic I/O IPCs. Using the tool described in Section 7 we can now reason about the maximal probability $\operatorname{Pr}\left(\diamond^{\leq t} s_{\mathcal{G}}^{\prime \prime}\right)$ to break the code within a pre-specified number $t$ of guesses. We consider here the set of all distributed schedulers as we obviously want that the guesser uses only local information to make its guesses. If we were to consider the set of all schedulers the maximum probability would be 1 for any time-bound as the guesser would immediately choose the correct code with probability 1 . Note that it does not make sense to consider strongly distributed schedulers for this case study as it never occurs that the I/O-IPCs $\mathcal{G}$ and $\mathcal{E}$ both have immediate actions enabled. In other words, the players act in turn.

\begin{tabular}{c|c|c|c|c|c|c|c|c|c|c}
\hline \multicolumn{2}{l}{ Settings } & \multicolumn{2}{l|}{ PMC } & \multicolumn{3}{l|}{ PARAM } & \multicolumn{2}{l}{ NLP } \\
\hline$n$ & $m$ & $t$ & $\# S$ & $\# T$ & $\# V$ & Time(s) & Mem(MB) & $\# V$ & Time(s) & $P r$ \\
\hline 2 & 2 & 2 & 197 & 248 & 36 & 0.0492 & 1.43 & 17 & 0.0973 & 0.750 \\
2 & 2 & 3 & 629 & 788 & 148 & 0.130 & 2.68 & 73 & 0.653 & 1.00 \\
3 & 2 & 2 & 1545 & 2000 & 248 & 0.276 & 5.29 & 93 & 1.51 & 0.625 \\
3 & 2 & 3 & 10953 & 14152 & 2536 & 39.8 & 235 & 879 & 1433 & 1.00 \\
2 & 3 & 2 & 2197 & 2853 & 279 & 0.509 & 6.14 & 100 & 2.15 & 0.556 \\
\hline
\end{tabular}

Table 1: Results of Mastermind Case Study

Results are given in Table 1. In addition to the model parameters $(n, m)$, the time bound $(t)$ and the result $(P r)$. We provide statistics for the various phases of the algorithm. For the unfolded PMC we gives the number of states $(\# S)$, transitions $(\# T)$, and variables $(\# V)$. For the PARAM tool we give the time needed to compute the polynomial, the memory required, and the number of variables that remain in the resulting polynomial. Finally we give the time needed for Matlab to optimize the polynomial provided by PARAM under the linear constraints that all scheduler decisions lie between 0 and 1 . For this case study we generated PMC models and linear constraints semi-automically given the parameters $n, m$, and $t$.

\subsection{Dining cryptographers}

The dining cryptographers problem is a classical anonymity problem [4]. The cryptographers must work together to deduce a particular piece of information using their local knowledge, but at the same time each cryptographers' local knowledge may not be discovered by the others. The problem is as follows: three cryptograpers have just finished dining in a restaurant when their waiter arrives to tell them their bill has been paid anonymously. The cryptographers now decide they wish to respect the anonimity of the payer, but they wonder if one of the cryptographers has paid or someone else. They resolve to use the following protocol to discover whether one of the cryptographers paid, without revealing which one. 
We depict part of the I/O-IPC models in Fig. 5. On the right-hand side we have the I/O-IPC $\mathcal{F}$ that simply decides who paid (actions $p_{i}$ ) and then starts the protocol. Each cryptographer has a probability of $\frac{1}{6}$ to have paid and there is a probability of $\frac{1}{2}$ that none of them has paid. On the left-hand side of Fig. 5 we see part of the I/O-IPC $\mathcal{P}_{\infty}$ for the first cryptographer. Each cryptographer flips a fair coin such that the others cannot see the outcome. In Fig. 5 we only show the case where cryptographer one flips heads. Each cryptographer now shows his coin to himself and his right-hand neighbour (actions $h_{i}$ for heads and $t_{i}$ for tails). This happens in a fixed order. Each cryptographer now knows the outcome of two coins (for instance, cryptographer one knows the outcome of his own coinflip and the outcome of that of cryptographer two). Now, again in a fixed order, they proclaim whether or not the two coins were the same or different (actions $s_{i}$ for same and $d_{i}$ for different). However, if a cryptographer has paid he or she will lie when proclaiming whether the two coins were identical or not. In Fig. 5 we show the case where cryptographer one has not paid, so he proclaims the truth. Now we have that if there is an even number of "different" proclamations, then all of the cryptographers told the truth and it is revealed that someone else paid. If, on the other hand, there is an odd number of "different" proclamations, one of the cryptographers must have paid the bill, but it has been shown that there is be no way for the other two cryptographers to know which one has paid. In our model the cryptographer first attempts to guess whether or not a cryptographer has paid (actions $c_{i}$ to guess that a cryptographer has paid, action $n_{i}$ if not). In case the cryptographer decides a cryptographer has paid, he guesses which one (action $g_{i, j}$ denotes that cryptographer $i$ guesses cryptographer $j$ has paid.
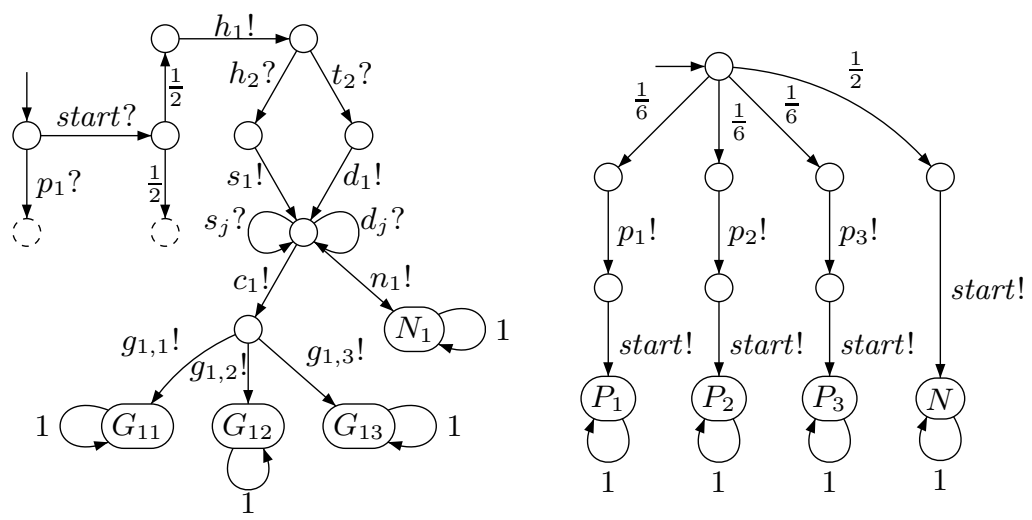

Fig. 5: Part of the I/O-IPC model $\mathcal{G}_{1}$ (left) of the first dining cryptographer and the I/O-IPC $\mathcal{F}$ (right) that probabilistically decides who has actually paid.

We can see that a "run" of the distributed I/O-IPC $\mathcal{C}=\mathcal{F}\left\|\mathcal{G}_{1}\right\| \mathcal{G}_{2} \| \mathcal{G}_{3}$ takes two time-units, since there is one probabilistic step to determine who paid and one probabilistic step where all coins are tossed simultaneously. We are interested in two properties of this algorithm: first, all cryptographers should be able to 
determine whether someone else has paid or not. We can express this property, for example for the first cryptographer, as a reachability probability property:

$$
P\left(\diamond^{\leq 2}\left\{P_{1}, P_{2}, P_{3}\right\} \times\left\{G_{11}, G_{12}, G_{13}\right\} \times S_{2} \times S_{3} \cup\{N\} \times\left\{N_{1}\right\} \times S_{2} \times S_{3}\right)=1 .
$$

Here $S_{2}$ and $S_{3}$ denote the complete state spaces of the second and third cryptographer I/O-IPCs. For the other cryptographers we find similar reachability probability properties. Secondly, we must check that the payer remains anonymous. This means that, in the case that a cryptographer pays, the other two cryptographers cannot guess this fact. We can formulate this as a conditional reachability probability:

$$
\frac{P\left(\diamond \leq 2\left\{P_{2}\right\} \times\left\{G_{12}\right\} \times S_{2} \times S_{3} \cup\left\{P_{3}\right\} \times\left\{G_{13}\right\} \times S_{2} \times S_{3}\right)}{P\left(\diamond \leq 2\left\{P_{2}, P_{3}\right\} \times S_{1} \times S_{2} \times S_{3}\right)}=\frac{1}{2} .
$$

I.e., the probability that the first cryptographer guesses correctly which other cryptographer has paid, under the condition that one of the other cryptographers has paid is one half.

\begin{tabular}{c|c|l|l|l|c|c|c|c}
\hline & \multicolumn{3}{l|}{ PMC } & \multicolumn{3}{l|}{ PARAM } & \multicolumn{2}{l}{ NLP } \\
\hline Property & $\# S$ & $\# T$ & $\# V$ & Time(s) & Mem(MB) & $\# V$ & Time(s) & $P r$ \\
\hline (4) & 294 & 411 & 97 & 9.05 & 4.11 & 24 & 0.269 & 1.00 \\
(5), top & 382 & 571 & 97 & 9.03 & 4.73 & 16 & 0.171 & 0.167 \\
(5), bottom & 200 & 294 & 97 & 8.98 & 4.14 & 0 & N/A & $1 / 3$ \\
\hline
\end{tabular}

Table 2: Results of Dining Cryptographers Case Study.

Table 2 shows the results for the dining cryptographers case study. We compute the conditional probability in (5) by computing the top and bottom of the fraction separately. We can see that both properties (4) and (5) are fulfilled. Table 2 also lists statistics on the tool performances and model sizes as described for Table 1 . Note especially that the third reachability probability was computed by directly by PARAM. I.e., this probability is independent of the scheduler decisions and PARAM was able to eliminate all variables.

\subsection{Randomized Scheduler Example}

For the class of strongly distributed schedulers it may be the case that the maximal or minimal reachability probability can not be attained by a deterministic scheduler, i.e., a scheduler that always chooses one action/component with probability one. As our final case study we use a small example of such an I/O-IPC as depicted by Fig. 4 in [10]. In this example the maximal reachability probability for deterministic strongly distributed schedulers is $\frac{1}{2}$, while there exists a randomized strongly distributed scheduler with reachability probability $\frac{13}{24}$.

Table 3 shows the result of applying our tool chain to this example. We see that we can find a scheduler with maximal reachability probability 0.545 , which is even greater than $\frac{13}{24}$. Note that we can express the maximal reachability probability as a time-bounded property because the example is acyclic. However, for this case, the result from Matlab depends on the initial assignment 
given to the solver. For certain initial assignments the solver returns a maximal probability of only 0.500 . This indicates that further investigation is required in the appropriate nonlinear programming tool for our algorithm.

\begin{tabular}{l|c|c|c|c|c|c|c}
\hline \multicolumn{3}{l|}{ PMC } & \multicolumn{3}{l|}{ PARAM } & \multicolumn{2}{l}{ NLP } \\
\hline$\# S$ & $\# T$ & $\# V$ & Time(s) & Mem(MB) & $\# V$ & Time(s) & Pr \\
\hline 13 & 23 & 12 & 0.00396 & 1.39 & 11 & 0.241 & $0.545^{5}$ \\
\hline
\end{tabular}

Table 3: Results of Randomized Scheduler Case Study.

\section{Conclusion}

In this paper we have presented an algorithm to compute maximal and minimal time-bounded reachability probabilities for I/O-IPCs under distributed schedulers or strongly distributed schedulers. The core principle of our algorithm is to reformulate the problem as a polynomial optimization problem under linear and, in the case of strongly distributed schedulers, non-linear constraints.

The main drawback of our approach is that the PMC induced in our algorithm grows exponentially with the size of our original model and the specified time-bound, as the state space of the PMC consists of all paths of the original model, up to a time-bound. However, no other algorithm exists that can compute properties of distributed models under (strongly) distributed schedulers.

In several areas improvements can be made. First, it can be investigated if special purpose algorithms can be used for the specific type of non-linear programming problems we encounter in our context. Secondly, the memory-usage may be optimized by using the fact that in our setting we see only polynomial function and do not make use of rational polynomial functions.

\section{References}

1. L. H. Ault. Das Mastermind-Handbuch. Ravensburger Buchverlag, 1982.

2. A. Bianco and L. de Alfaro. Model checking of probabalistic and nondeterministic systems. In Proceedings of the 15th Conference on Foundations of Software Technology and Theoretical Computer Science, pages 499-513. Springer-Verlag, 1995.

3. H. Boudali, P. Crouzen, and M. Stoelinga. A Compositional Semantics for Dynamic Fault Trees in Terms of Interactive Markov Chains. In ATVA, pages 441-456, 2007.

4. D. Chaum. The dining cryptographers problem: Unconditional sender and recipient untraceability. Journal of Cryptology, 1(1):65-75, 1988.

5. N. Coste, H. Garavel, H. Hermanns, R. Hersemeule, Y. Thonnart, and M. Zidouni. Quantitative Evaluation in Embedded System Design: Validation of Multiprocessor Multithreaded Architectures. In DATE, pages 88-89, 2008.

6. N. Coste, H. Hermanns, E. Lantreibecq, and W. Serwe. Towards Performance Prediction of Compositional Models in Industrial GALS Designs. In Computer Aided Verification, pages 204-218, 2009.

7. C. Daws. Symbolic and Parametric Model Checking of Discrete-Time Markov Chains. In ICTAC, pages 280-294, 2004.

8. P. Gill, W. Murray, and M. Wright. Practical Optimization. Academic Press, London, 1981.

\footnotetext{
${ }^{5}$ For certain settings, Matlab reports a maximal probability of 0.500
} 
9. S. Giro and P. R. D'Argenio. Quantitative model checking revisited: Neither decidable nor approximable. In J.-F. Raskin and P. S. Thiagarajan, editors, FORMATS, volume 4763 of Lecture Notes in Computer Science, pages 179-194. Springer, 2007.

10. S. Giro and P. R. D'Argenio. On the expressive power of schedulers in distributed probabilistic systems. Electronic Notes in Theoretical Computer Science, 253(3):45-71, 2009.

11. E. M. Hahn, H. Hermanns, B. Wachter, and L. Zhang. PARAM: A Model Checker for Parametric Markov Models. In $C A V, 2010$.

12. E. M. Hahn, H. Hermanns, and L. Zhang. Probabilistic Reachability for Parametric Markov Models. STTT, 2010.

13. S. Han. A globally convergent method for nonlinear programming. Journal of Optimization Theory and Applications, 22, 1977.

14. H. Hermanns. Interactive Markov Chains: The Quest for Quantified Quality, volume 2428 of Lecture Notes in Computer Science. Springer-Verlag, 2002.

15. L. Zhang and M. R. Neuhäußer. Model checking interactive markov chains. In TACAS, pages 53-68, 2010. 


\section{A Proofs}

\section{A.1 Proof of Lemma 1}

Proof. For (i) and (ii) set the assignment $\zeta$, respectively the distributed scheduler $\eta$ such that for $1 \leq i \leq \# \mathcal{C}: \zeta\left(y_{\sigma}^{i}\right)=\mathcal{I}(\sigma)\left(\mathcal{P}_{i}\right)$ if $A_{\text {last }\left(\sigma\left[\mathcal{P}_{i}\right]\right), \mathcal{P}_{i}}^{\text {en }} \neq \varnothing$ and $\zeta\left(x_{\sigma\left[\mathcal{P}_{i}\right]}^{a}\right)=\eta_{\mathcal{P}_{i}}\left(\sigma\left[\mathcal{P}_{i}\right]\right)(a)$ if $a \in A_{\text {last }\left(\sigma\left[\mathcal{P}_{i}\right]\right), \mathcal{P}_{i}}^{\text {en }}$ This gives indentical mappings from assignments to distributed schedulers and back for (i) and (ii), which means we can prove both simultaneously.

For a distributed scheduler $\eta$ and its associated assignment $\zeta$, we now show that $P_{\eta}\left(\sigma^{\uparrow}\right)=\zeta(\mathbf{P}(\hat{s}, \sigma))$ by induction on the length of $\sigma$ : For paths of length 0 we have that $P_{\eta}\left(\hat{s}^{\uparrow}\right)=1=\zeta\left(\mathbf{P}_{0}(\hat{s}, \hat{s})\right)$ and $P_{\eta}\left(s^{\uparrow}\right)=0=\zeta\left(\mathbf{P}_{0}(\hat{s}, s)\right)$, for $s \neq \hat{s}$. For the inductive step, let the induction hypothesis (IH) be the following. Given a path $\sigma \in S_{\mathcal{C}}^{t}$ of length $k>0$, we have for any path $\sigma^{\prime}$ length $k-1$, that: $P_{\eta}\left(\sigma^{\prime}\right)=\zeta\left(\mathbf{P}_{k-1}\left(\hat{s}, \sigma^{\prime}\right)\right)$. By case distinction we now have:

1. For $\operatorname{last}(\sigma)$ vanishing we have:

$$
\begin{aligned}
P_{\eta}\left(\sigma^{\uparrow}\right) & \stackrel{D e f}{=}{ }^{9} \sum_{\sigma=\sigma^{\prime} a s} P_{\eta}\left(\sigma^{\prime}\right) \cdot \eta\left(\sigma^{\prime}\right)(a) \stackrel{I H}{=} \sum_{\sigma=\sigma^{\prime} a s} \zeta\left(\mathbf{P}_{k-1}\left(\hat{s}, \sigma^{\prime}\right)\right) \cdot \eta\left(\sigma^{\prime}\right)(a) \\
& \stackrel{\text { Def } 7}{=} \sum_{\sigma=\sigma^{\prime} a s} \zeta\left(\mathbf{P}_{k-1}\left(\hat{s}, \sigma^{\prime}\right)\right) \cdot \sum_{i=1, e(i, a, \sigma)}^{n} \mathcal{I}(\sigma)\left(\mathcal{P}_{i}\right) \cdot \eta_{\mathcal{P}_{i}}\left(\sigma\left[\mathcal{P}_{i}\right]\right)(a) \\
& \stackrel{D e f}{=} \sum_{\sigma=\sigma^{\prime} a s} \zeta\left(\mathbf{P}_{k-1}\left(\hat{s}, \sigma^{\prime}\right)\right) \cdot \zeta\left(\mathbf{P}\left(\sigma^{\prime}, \sigma\right)\right)=\zeta\left(\mathbf{P}_{k}(\hat{s}, \sigma)\right) .
\end{aligned}
$$

2. For last $(\sigma)$ tangible we have: $P_{\eta}\left(\sigma^{\uparrow}\right) \stackrel{\text { Def }}{=}{ }^{9} \sum_{\sigma=\sigma^{\prime} \mu s} P_{\eta}\left(\sigma^{\uparrow}\right) \cdot \mu(s) \stackrel{\text { Def }}{\overline{\overline{I H}}} 11$ $\sum_{\sigma=\sigma^{\prime} \mu s} \zeta\left(\mathbf{P}_{k-1}\left(\hat{s}, \sigma^{\prime}\right)\right) \cdot \zeta\left(\mathbf{P}\left(\sigma^{\prime}, \sigma\right)\right)=\zeta\left(\mathbf{P}_{k}(\hat{s}, \sigma)\right)$.

\section{A.2 Proof of Theorem 1}

Proof. For a distributed scheduler $\eta$ with associated assignment $\zeta$, as defined in Lemma 1, we have $P_{\eta}\left(\nabla^{\leq t} \mathcal{G}\right)=P_{\eta}\left(\bigcup\left\{\sigma^{\uparrow} \mid \mathrm{t}(\sigma) \leq t\right.\right.$ and $\left.\left.\operatorname{last}(\sigma) \in \mathcal{G}\right\}\right)$. Now let the set of paths $\bar{S} \subset S_{\mathcal{C}}^{t}$ that end in a state in $\mathcal{G}$, but do not pass through $\mathcal{G}$ under way. It is obvious that the cylinders induced by these paths do not overlap and that their union is the set of all paths that reach $\mathcal{G}$ within $t$ time-units. We then have: $P_{\eta}\left(\diamond^{\leq t} \mathcal{G}\right)=P_{\eta}\left(\bigcup\left\{\sigma^{\uparrow} \mid \sigma \in \bar{S}\right\}\right)=\sum_{\sigma \in \bar{S}} P_{\eta}\left(\sigma^{\uparrow}\right)=$ $\sum_{\sigma \in \bar{S},|\sigma|=k} \zeta\left(\mathbf{P}_{k}(\hat{s}, \sigma)\right)=\zeta\left(\sum_{\sigma \in \bar{S},|\sigma|=k} \mathbf{P}_{k}(\hat{s}, \sigma)\right)=\zeta(\mathbf{P}(\diamond \bar{S}))$. The last equality stems from the fact that a path $\sigma$ of length $k$ can only be reached for the first time after exactly $k$ steps in $\mathcal{D}$.

\section{A.3 Proof of Theorem 2}

Proof. We associate strongly distributed schedulers $\eta$ to assignments $\zeta$ following Lemma 1. For the extra variables in $V_{z}$ we choose $\zeta\left(z_{\sigma\left[\mathcal{P}_{i}\right], \sigma\left[\mathcal{P}_{j}\right]}^{i, j}\right)$ equals $\zeta\left(\frac{y_{\sigma}^{i}}{y_{\sigma}^{i}+y_{\sigma}^{j}}\right)$ if $\zeta\left(y_{\sigma}^{i}+y_{\sigma}^{j}\right)>0$ and 1 otherwise. Note that the value 1 is chosen arbitrarily here. 
To prove Theorem 2 we must now show that any assignment that satisfies (3) is associated to a strongly distributed scheduler and that any strongly distributed scheduler is associated to an assigment that satisfies (3).

First, notice that for a path $\sigma$ ending in a vanishing state and distinct I/OIPCs $\mathcal{P}_{i}$ and $\mathcal{P}_{j}$ that have immediate actions enabled after $\sigma$, we have that if $\zeta\left(y_{\sigma}^{i}\right)=0=\zeta\left(y_{\sigma}^{j}\right)$ then $z_{\sigma\left[\mathcal{P}_{i}\right], \sigma\left[\mathcal{P}_{j}\right]}^{i, j}\left(y_{\sigma}^{i}+y_{\sigma}^{j}\right)=y_{\sigma}^{i}$ holds, regardless the value of $\zeta\left(z_{\sigma\left[\mathcal{P}_{i}\right], \sigma\left[\mathcal{P}_{j}\right]}^{i, j}\right)$.

Now, consider an assignment $\zeta$ with associated distributed scheduler $\eta$, and suppose we find two paths $\sigma, \sigma^{\prime}$ as above with $\zeta\left(y_{\sigma}^{i}+y_{\sigma}^{j}\right) \neq 0 \neq \zeta\left(y_{\sigma^{\prime}}^{i}+y_{\sigma^{\prime}}^{j}\right)$, $\sigma\left[\mathcal{P}_{i}\right]=\sigma^{\prime}\left[\mathcal{P}_{i}\right]$ and $\sigma\left[\mathcal{P}_{j}\right]=\sigma^{\prime}\left[\mathcal{P}_{j}\right]$. Then $(3)$ gives us that:

$$
\zeta\left(\frac{y_{\sigma}^{i}}{y_{\sigma}^{i}+y_{\sigma}^{j}}\right)=\zeta\left(z_{\sigma\left[\mathcal{P}_{i}\right], \sigma\left[\mathcal{P}_{j}\right]}^{i, j}\right)=\zeta\left(z_{\sigma^{\prime}\left[\mathcal{P}_{i}\right], \sigma^{\prime}\left[\mathcal{P}_{j}\right]}^{i, j}\right)=\zeta\left(\frac{y_{\sigma^{\prime}}^{i}}{y_{\sigma^{\prime}}^{i}+y_{\sigma^{\prime}}^{j}}\right)
$$

Since $\zeta\left(y_{\sigma}^{i}\right)$ corresponds to $\mathcal{I}(\sigma)\left(\mathcal{P}_{i}\right)$ in $\eta$, and similarly for $y_{\sigma}^{j}, y_{\sigma^{\prime}}^{i}, y_{\sigma^{\prime}}^{j}$, we have that now Equation. (1) holds for $\eta$, which means that $\eta$ is indeed strongly distributed. Since we have a one-to-one correspondence between distributed schedulers and assigments, this also proves the reverse, that the assigment associated to a strongly distributed scheduler satisfies (3). The remainder of the proof follows that of Theorem 1 . 